\title{
HOMOLOGICAL PISOT SUBSTITUTIONS AND EXACT REGULARITY
}

\author{
MARCY BARGE, HENK BRUIN, LESLIE JONES, AND LORENZO SADUN
}

\begin{abstract}
We consider one-dimensional substitution tiling spaces where the dilatation (stretching factor) is a degree $d$ Pisot number, and the first rational Čech cohomology is $d$-dimensional. We construct examples of such "homological Pisot" substitutions whose tiling flows do not have pure discrete spectra. These examples are not unimodular, and we conjecture that the coincidence rank must always divide a power of the norm of the dilatation. To support this conjecture, we show that homological Pisot substitutions exhibit an Exact Regularity Property (ERP), in which the number of occurrences of a patch for a return length is governed strictly by the length. The ERP puts strong constraints on the measure of any cylinder set in the corresponding tiling space.
\end{abstract}

\section{INTRODUCTION}

Versions of the Pisot Conjecture occur in number theory (numeration systems, $\beta$ expansions), discrete geometry, dynamical systems (construction of Markov partitions, arithmetical coding of hyperbolic toral automorphisms), and physics (spectral properties of materials with quasi-periodic atomic structure) - see the survey [BS]. In all of these settings there is an underlying substitution and in this context a standard version of the conjecture is

Pisot Conjecture: The $\mathbb{R}$-action (tiling flow) on the tiling space associated with a one-dimensional substitution of unimodular and irreducible Pisot type has pure discrete spectrum.

It is known that any such action has a non-trivial discrete part in its spectrum, see [BT], and the conjecture is known to be true in the case of a substitution on two letters, see [BD2, $\mathrm{HS}$. One of the reasons for interest in this conjecture is that the $\mathbb{R}$-action on a substitution tiling space has a pure discrete spectrum (also known as pure point spectrum) if and only if a one-dimensional quasicrystal, whose atoms are

2000 Mathematics Subject Classification. Primary: 37B50, 54H20, Secondary: 11R06, 37B10, $55 \mathrm{~N} 05,55 \mathrm{~N} 35,52 \mathrm{C} 23$.

Key words and phrases. Pisot Conjecture, Substitution, Tiling Space, Kronecker Flow, Inverse Limit Space. 
arranged according to the pattern of any tiling in the tiling space, has pure discrete diffraction spectrum, see [Dw, LMS].

A substitution $\phi$ is a function from a finite alphabet $\mathcal{A}$ into the collection of finite nonempty words from $\mathcal{A}$, and extends by concatenation to a map on finite or infinite words. The abelianization of $\phi$ is the matrix $\mathbf{A}=\mathbf{A}_{\phi}$ with $i j$-th entry equal to the number of $i$ 's in $\phi(j)$. The substitution $\phi$ is primitive if the entries of $\mathbf{A}^{m}$ are strictly positive for some $m \geq 1$. In this case $\mathbf{A}$ has a simple, positive, Perron-Frobenius eigenvalue $\lambda=\lambda_{\phi}$ which we will call the dilatation of $\phi$.

Definition 1 (Pisot Substitution). A Pisot number is an algebraic integer greater than 1 , all of whose algebraic conjugates lie strictly inside the unit circle. If the dilatation of a primitive substitution is a Pisot number of algebraic degree $d$, then we say that the substitution is Pisot of degree $d$.

Let $p(x)=x^{d}+a_{d-1} x^{d-1}+\cdots+a_{0}$ be the minimal polynomial of the dilatation. The norm of the dilatation is the product of the dilatation and its algebraic conjugates and equals $(-1)^{d} a_{0}$. We call $a_{0}$ the constant coefficient of the dilatation.

Definition 2 (Irreducible Substitution). The minimal polynomial $p(x)$ always divides the characteristic polynomial of the abelianization of $\phi$. If the two polynomials are equal, then we say that the substitution is irreducible.

A substitution $\phi$ generates a collection of bi-infinite allowed words, namely those $\bar{w}=\ldots w_{-1} w_{0} w_{1} \ldots$ for which each finite subword $w_{i} \ldots w_{i+j}$ is a subword of $\phi^{m}(a)$ for some $m \in \mathbb{N}$ and $a \in \mathcal{A}$. To each $a \in \mathcal{A}$ we associate a prototile $P_{a}$ whose length equals the $a$-th component of the left Perron-Frobenius eigenvector of $\mathbf{A}_{\phi}$. To each allowed word $\bar{w}$ we can associate continuously many tilings $\mathbf{T}$, replacing each letter $w_{i}$ by a copy of the corresponding prototile, and putting an origin somewhere in the obtained concatenation of tiles. The tiling space, $\Omega_{\phi}$, is the collection of all such tiles, and it carries a natural tiling flow, $\mathbf{T}=\left\{T_{i}\right\} \mapsto \mathbf{T}-t:=\left\{T_{i}-t\right\}$.

When studying substitutions, it is natural to require that the substitution be irreducible and Pisot. However, irreducibility is not a natural condition for tiling spaces, insofar as different substitutions may give rise to the same tiling space. The substitutions $1 \mapsto 21,2 \mapsto 1$ and $1 \mapsto 32,2 \mapsto 31,3 \mapsto 2$ both generate the Fibonacci tiling, but the first is irreducible while the second is not. We therefore introduce a topological condition, noting that if two one-dimensional substitution tiling spaces are homeomorphic, then the tiling flow on one of them has pure discrete spectrum if and only if the tiling flow on the other does. (This is a consequence of the rigidity result of $[\mathrm{BSw}$.$) We also consider relaxing the requirement of unimodularity, as there are$ many instances where pure discreteness is known to hold when the Pisot dilatation has 
norm other than \pm 1 (in particular, for a large family of substitutions associated with $\beta$-expansions - see [BBK]).

Definition 3 (Homological Pisot Substitution). Let $\phi$ be a degree d Pisot substitution. If the dimension of the first rational Čech cohomology of the tiling space is $d$, then we say that the substitution is homological Pisot of degree $d$.

The dilatation being a Pisot number is a necessary and sufficient condition for the tiling flow spectrum to have a discrete component, see [So1]. However, it is easy to find examples of substitutions with Pisot dilatations whose spectra contain a continuous component. The simplest example is the Thue-Morse substitution $1 \mapsto 12,2 \mapsto 21$. To eliminate the continuous spectrum, we must constrain the system further, either through a combinatorial condition (irreducibility) or through a topological condition (dimension of the first cohomology).

In this paper we study the consequences of a substitution being homological Pisot, including (but not limited to) the question of whether the tiling flow associated with such a substitution must have pure discrete spectrum. Note that the homological Pisot condition is neither stronger nor weaker than the irreducible Pisot condition. It is easy to construct homological Pisot substitutions that are not irreducible - just rewrite any homological Pisot substitution in terms of collared tiles, see [AP]. It is also easy to find examples of irreducible Pisot substitutions whose first cohomology has dimension greater than $d$ (e.g. the substitution, $1 \mapsto 21112,2 \mapsto 121$, is an irreducible degree 2 Pisot substitution with an asymptotic cycle, whose tiling space has 3-dimensional first cohomology). Nevertheless, the two notions are closely related: an irreducible Pisot substitution that does not have asymptotic cycles must be homological Pisot. Also, if the characteristic polynomial of the abelianization of a homological Pisot substitution does factor over $\mathbb{Z}$, say as $p(x) q(x)$, with $p(x)$ the minimal polynomial of the dilatation, then all roots of $q(x)$ are zero or roots of unity.

The Coincidence Rank Conjecture. Every one-dimensional substitution tiling space has a maximal equicontinuous factor consisting of a Kronecker flow on a torus or solenoid. The continuous map factoring the tiling flow onto its maximal equicontinuous factor is called geometric realization and also factors the substitution homeomorphism onto a hyperbolic automorphism of the torus or solenoid. Geometric realization has the following properties $([\mathrm{BK},[\mathrm{BBK}])$ : it is nontrivial if and only if the substitution is Pisot and, if the substitution is Pisot, then geometric realization is boundedly finite-to-one and almost everywhere $c r$-to-one for some positive integer $\mathrm{cr}$ called the coincidence rank of the substitution. Furthermore, the tiling flow has pure discrete spectrum if 
and only if geometric realization is a.e. 1-to-1 (that is, if $c r=1$ ), in which case geometric realization is a continuous measurable isomorphism from the tiling flow onto a Kronecker action.

We can obtain a topological version of the Pisot Conjecture by dropping the unimodular assumption (i.e., norm $= \pm 1$ ) and replacing "irreducible Pisot" by "homological Pisot", leading to the conjecture:

The tiling flow of a homological Pisot substitution has pure discrete spectrum.

This conjecture is false. In the last section of this paper we give several examples of homological Pisot substitutions with coincidence rank 3 and norm divisible by 3 , and in fact, such examples generalize to every algebraic degree.

Theorem 1. There are homological Pisot substitutions of every algebraic degree whose tiling flows do not have pure discrete spectrum.

That the coincidence rank in these examples divides the norm is not a coincidence and we are led to the following conjecture.

Coincidence Rank Conjecture: The coincidence rank of a homological Pisot substitution divides a power of the norm. In particular, the tiling flow of a unimodular homological Pisot substitution has pure discrete spectrum.

Theorem 2. The Coincidence Rank Conjecture is true if the degree of the substitution is one.

Exact Regularity. A patch $\mathbf{P}$ of a tiling $\mathbf{T}$ is a collection of contiguous tiles of $\mathbf{T}$ and the support of a patch is the union of all the tiles in the patch. A vertex is any boundary point of two adjacent tiles in a tiling or patch.

The tile lattice, $\Gamma$, for the tiling space $\Omega_{\phi}$ is the additive subgroup of $\mathbb{R}$ generated by the lengths of the tiles of $\Omega_{\phi}$. A return length for the tiling space $\Omega_{\phi}$ is a number $L>0$, such that $L$ is the distance between corresponding points in separate occurrences of the same tile type in $\mathbf{T}$ for some $\mathbf{T} \in \Omega_{\phi}$. The return lattice for $\Omega_{\phi}$ is the subgroup of the tile lattice $\Gamma$ generated by $\left\{\lambda^{-i} L: L\right.$ is a return length and $\left.i \geq 0\right\} \cap \Gamma$, where $\lambda$ is the dilatation of $\phi$. These objects are called lattices, despite typically being dense in $\mathbb{R}$, as they are projections to $\mathbb{R}$ of lattices in $\mathbb{Z}^{d}$, where $d$ is the number of letters in the substitution. The tile and return lattices for a 'proper' substitution are the same, and a power of any primitive substitution can be 'rewritten' to a proper substitution see e.g. [BD1.

Definition 4 (The Exact Regularity Property). Let $\lambda$ denote the dilatation of the substitution $\phi$ and let $L>0$ be in the return lattice for $\Omega_{\phi}$. We say that the tiling space $\Omega_{\phi}$ exhibits the Exact Regularity Property (ERP) if for each patch $\mathbf{P}$ of any 
tiling in $\Omega_{\phi}$ there is a length $L^{\prime}$ and a linear functional $\mathbf{N}_{\mathbf{P}}: \mathbb{Q}(\lambda) \rightarrow \mathbb{Q}$ such that: if $\mathbf{Q}$ and $\mathbf{Q}+\tau$ are patches that occur in any tiling $\mathbf{T}$ in $\Omega_{\phi}$ and $\mathbf{Q}$ has a vertex $x_{0}$ with $\left[x_{0}-L^{\prime}, x_{0}+L^{\prime}\right]$ contained in the support of $\mathbf{Q}$, then the number of occurrences of $\mathbf{P}$ in $\mathbf{T}$ between $x_{0}$ and $x_{0}+\tau$ is exactly $\mathbf{N}_{\mathbf{P}}(\tau / L)$. That is, if $x_{1}$ is a vertex of $\mathbf{P}$, then $\left\{t: 0 \leq t<\tau\right.$ and $\left.\mathbf{P}+x_{0}-x_{1}+t \subset \mathbf{T}\right\}$ has cardinality $\mathbf{N}_{\mathbf{P}}(\tau / L)$.

Theorem 3 (Exact Regularity). Homological Pisot substitution spaces exhibit the Exact Regularity Property. Moreover, given any patch $\mathbf{P}$, if $\mathbf{N}_{\mathbf{P}}$ is expressed in the form $\mathbf{N}_{\mathbf{P}}\left(\sum_{i=0}^{d-1} c_{i} \lambda^{i}\right)=\sum_{i=0}^{d-1} \alpha_{i} c_{i}$, with $\alpha_{i}, c_{i} \in \mathbb{Q}$, then $\alpha_{i} \in \mathbb{Z}\left[1 / a_{0}\right]$ for $i=0, \ldots, d-1$, where $a_{0}$ is the constant coefficient of $\lambda$.

Theorem 4. Any Pisot substitution that exhibits the Exact Regularity Property is a homological Pisot substitution.

If $\phi$ is irreducible Pisot, then the tiling flow $\mathbf{T}=\left\{T_{i}\right\} \mapsto \mathbf{T}-t:=\left\{T_{i}-t\right\}$ preserves a unique measure $\mu$. Using the ERP, we derive restrictions on the measures of measurable sets in the tiling space.

Theorem 5. Let $\phi$ be a homological Pisot substitution of algebraic degree $d$ and constant coefficient $a_{0}$. Suppose that the tile lattice and the return lattice for $\phi$ are the same. If some finite disjoint union of cylinder sets in the tiling space $\Omega_{\phi}$ has rational measure $n / m$, with $n$ and $m$ relatively prime, then $m$ divides $d \cdot a_{0}^{k}$ for some positive integer $k$.

If $d=1$, it is possible to construct a disjoint union of cylinder sets of measure $1 / \mathrm{cr}$ (Proposition 3). Theorem 5 then implies Theorem 2.

It is even possible to extend the idea of the ERP to one-dimensional tiling spaces that do not come from a substitution, ([Sa2]). If the first rational Cech cohomology of a tiling space $\Omega$ is $k$-dimensional, then we can find $k$ different collections of patches (call them patches of type $1,2, \ldots, k$ ), such that for any other patch $\mathbf{P}$ there is a length $L^{\prime}$ with the following property: If $\mathbf{Q}$ and $\mathbf{Q}+\tau$ are patches that occur in any tiling $\mathbf{T}$ in $\Omega_{\phi}$ and $\mathbf{Q}$ has a vertex $x_{0}$ with $\left[x_{0}-L^{\prime}, x_{0}+L^{\prime}\right]$ contained in the support of $\mathbf{Q}$, then the number of occurrences of $\mathbf{P}$ in $\mathbf{T}$ between $x_{0}$ and $x_{0}+\tau$ is a rational linear function of $n_{1}, \ldots, n_{k}$, where $n_{i}$ is the number of occurrences of patches of type $i$ between $x_{0}$ and $x_{0}+\tau$. When the tiling space comes from a substitution and $k$ is the algebraic degree of the dilatation, then the $i$-th class of patches is associated with tiles of length $L \lambda^{i-1}$.

Organization of the paper. In Section 2, we develop necessary background and notation. In Section 3, we explore the ERP and prove Theorems 3, 4 and 5. The proofs of these theorems do not use the fact that the dilatation is a Pisot number, and 
the results of Section 3 apply to all substitutions for which the dimension of the first Cech cohomology equals the algebraic degree of the dilatation.

In Section 4, we study $d=1$ homological Pisot substitutions and prove Theorem 2 . We also show, regardless of the norm:

Theorem 6. If $\phi$ is a homological Pisot substitution with $d=1$, then the coincidence rank is not 2.

Finally, in Section 5 we show how to construct homological Pisot substitutions of arbitrary $d$ with $c r=3$, thereby proving Theorem 1.

\section{BACKGROUND}

A substitution is a function $\phi: \mathcal{A} \rightarrow \mathcal{A}^{*}$ from a finite alphabet $\mathcal{A}$ into the collection $\mathcal{A}^{*}$ of finite nonempty words in $\mathcal{A}$. A substitution extends by concatenation to a map on finite or infinite words and can be iterated: $\phi^{m}$ will stand for $\phi \circ \phi \circ \cdots \circ \phi, m$ times. The abelianization or substitution matrix of $\phi$ is the matrix $\mathbf{A}=\mathbf{A}_{\phi}$ with $i j$-th entry equal to the number of $i$ 's in $\phi(j)$. The substitution $\phi$ is primitive if the entries of $\mathbf{A}^{m}$ are strictly positive for some $m \geq 1$. In this case $\mathbf{A}$ has a simple, positive, PerronFrobenius eigenvalue $\lambda=\lambda_{\phi}$ which we will call the dilatation of $\phi$. If $\phi$ is primitive, we will use $\omega^{l}$ and $\omega^{r}$ to denote left and right positive eigenvectors of $\mathbf{A}$.

To construct the tiling space associated with the primitive substitution $\phi$ we first form the collection $\mathbf{X}_{\phi} \subset \mathcal{A}^{\mathbb{Z}}$ consisting of all bi-infinite allowed words for $\phi: \bar{w}=$ $\ldots w_{-1} w_{0} w_{1} \ldots \in \mathbf{X}_{\phi}$ if and only if for each $i \in \mathbb{Z}$, and each $j \geq 0$, there is an $m \in \mathbb{N}$ and $a \in \mathcal{A}$ so that the word $w_{i} \ldots w_{i+j}$ is a factor (subword) of $\phi^{m}(a)$. If $\mathcal{A}=\{1, \ldots, n\}$ and the left eigenvector of $\mathbf{A}$ is $\omega^{l}=\left(\omega_{1}, \ldots, \omega_{n}\right)$, the intervals $P_{i}=\left[0, \omega_{i}\right], i=1, \ldots, n$, are called prototiles (in case $\omega_{i}=\omega_{j}$ for $i \neq j$, we label $P_{i}$ and $P_{j}$ so as to make them distinct). The tiling space, $\Omega_{\phi}$, associated with $\phi$ is the collection of all tilings of $\mathbb{R}$ by translates of prototiles following patterns of allowed bi-infinite words.

A patch $\mathbf{P}$ of a tiling $\mathbf{T}$ is a subcollection of contiguous tiles of $\mathbf{T}$ and the support of a patch is the union of all the tiles in the patch. We will denote the diameter of the support of $\mathbf{P}$ by $|\mathbf{P}|$ and call this the length of $\mathbf{P}$.

There is a natural tiling flow, $\mathbf{T}=\left\{T_{i}\right\} \mapsto \mathbf{T}-t:=\left\{T_{i}-t\right\}$. We put a metric on $\Omega_{\phi}$ with the property that $\mathbf{T}$ and $\mathbf{T}^{\prime}$ are close if small translates of $\mathbf{T}$ and $\mathbf{T}^{\prime}$ agree in a large neighborhood of the origin. If $\phi$ is primitive then, with this metric, $\Omega_{\phi}$ is a continuum (compact and connected) and the tiling flow is minimal and uniquely ergodic.

The substitution $\phi$ is aperiodic provided there are no flow-periodic tilings in $\Omega_{\phi}$. There is also a $\mathbb{Z}$-action on $\Omega_{\phi}$ induced by substitution. To define this, suppose that 
$i \in \mathcal{A}$ and $\phi(i)=i_{1} \cdots i_{k}$. Define $\Phi$ on prototiles by

$$
\Phi\left(P_{i}\right):=\left\{P_{i_{1}}, P_{i_{2}}+\omega_{i_{1}}, \ldots, P_{i_{k}}+\omega_{i_{1}}+\omega_{1_{2}}+\cdots+\omega_{i_{k-1}}\right\} .
$$

Extend this to tiles by $\Phi\left(P_{i}+t\right):=\Phi\left(P_{i}\right)+\lambda t$, and extend to tilings by $\Phi\left(\left\{T_{i}\right\}\right):=$ $\cup \Phi\left(T_{i}\right)$. As long as $\phi$ is primitive and aperiodic, $\Phi$ is a homeomorphism, see [M], So2].

We also recall the machinery of pattern-equivariant cohomology with rational coefficients from [Kel, $\mathrm{KP}$, Sa1]. A tiling $\mathbf{T} \in \Omega_{\phi}$ gives the real line the structure of a CW complex, with the vertices serving as 0-cells and the tiles serving as 1-cells.

Definition 5. A rational 0-cochain is said to be pattern-equivariant with radius $R$ if, whenever $x$ and $y$ are vertices of $\mathbf{T}$ and the radius $R$ neighborhoods $\mathbf{B}_{R}[\mathbf{T}-x]=\mathbf{B}_{R}[\mathbf{T}-$ $y]$, the cochain takes the same values at $x$ and $y$. $A$ 0-cochain is pattern-equivariant if it is pattern-equivariant with radius $R$ for some finite $R$. Pattern-equivariant 1cochains are defined similarly - their values on a 1-cell depend only on the pattern of the tiling out to a fixed finite distance around that 1-cell.

If $\beta$ is a rational pattern-equivariant 0 -cochain, its coboundary, $\delta(\beta)$, is a rational pattern-equivariant 1-cochain, and we define the rational first pattern-equivariant cohomology of $\mathbf{T}$ to be the cokernel of the coboundary map $\delta$. A priori this would seem to depend on $\mathbf{T}$, but this cohomology is the same for all $\mathbf{T} \in \Omega_{\phi}$ and is isomorphic to $\check{H}^{1}\left(\Omega_{\phi}, \mathbb{Q}\right)$, see [Kel, KP, Sa1].

Finally, we recall a procedure for computing the first Čech cohomology of a tiling space, using the machinery of [BD3]. To each primitive one-dimensional substitution $\phi$ on $n$ letters, one can associate a graph $G$. This graph has two kinds of edges. There is one edge $e_{i}$ for each letter $a_{i}$ of the alphabet, and one edge $v_{i j}$ for each two-letter word $a_{i} a_{j}$. The edge $e_{i}$ has length $\omega_{i}-\epsilon$ and represents the bulk of a tile of type $a_{i}$, while $v_{i j}$ has length $\epsilon$ and represents the transition from the end of a tile of type $i$ to the beginning of a tile of type $j$. We identify the end of $e_{i}$ with the beginning of $v_{i j}$, and the end of $v_{i j}$ with the beginning of $e_{j}$. There is also a sub-complex $G_{0}$ obtained from just the $v$ edges.

After applying a small homotopy, we may assume that substitution maps $G_{0}$ to itself. If $\phi\left(a_{i}\right)$ ends with $a_{k}$, and if $\phi\left(a_{j}\right)$ begins with $a_{\ell}$, then $\phi\left(v_{i j}\right)=v_{k \ell}$. Let $G_{0}^{E R}$ be the eventual range of $G_{0}$ under this map. Since substitution permutes the edges of $G_{0}^{E R}$, we can replace $\phi$ with a power that fixes each edge of $G_{0}^{E R}$. Then there is an exact sequence, BD3.

$$
0 \rightarrow \tilde{H}^{0}\left(G_{0}^{E R}\right) \rightarrow \lim _{\longrightarrow} \mathbf{A}^{T} \rightarrow \check{H}^{1}\left(\Omega_{\phi}\right) \rightarrow H^{1}\left(G_{0}^{E R}\right) \rightarrow 0
$$

that computes $\check{H}^{1}\left(\Omega_{\phi}\right)$. Furthermore, each map in this exact sequence commutes with substitution, so the image of $\tilde{H}^{0}\left(G_{0}^{E R}\right)$ lies in the +1 eigenspace of $\mathbf{A}^{T}$. Since the dilatation and its algebraic conjugates are all eigenvalues of $\mathbf{A}^{T}$, the dimension of 
$\check{H}^{1}\left(\Omega_{\phi}, \mathbb{Q}\right)$ is at least $d$, and equals $d$ only if three conditions are met: (1) the only eigenvalues of $\mathbf{A}^{T}$ are $0,1, \lambda$, and the algebraic conjugates of $\lambda,(2)$ the algebraic multiplicity of the eigenvalue 1 is one less than the number of components of $G_{0}^{E R}$, and (3) $G_{0}^{E R}$ has no loops.

\section{ExaCt REgularity}

Given a substitution $\phi$ with dilatation $\lambda$ of degree $d$, let us select a return length $L$. Then for every (by minimality of the flow) $\mathbf{T} \in \Omega_{\phi}, \mathbf{T}$ and $\mathbf{T}-L$ have nonempty intersection. It follows that for any $L^{\prime}$, there exists a $k$ such that $\phi^{k}(\mathbf{T})$ and $\phi^{k}(\mathbf{T})-\lambda^{k} L$, and hence $\mathbf{T}$ and $\mathbf{T}-\lambda^{k} L$, intersect in a patch of length at least $L^{\prime}$.

As $1, \lambda, \lambda^{2}, \ldots, \lambda^{d-1}$ are linearly independent over $\mathbb{Q}$, the length of each patch $\mathbf{P}$ can be expressed uniquely in the form $|\mathbf{P}|=L \sum_{i=0}^{d-1} c_{i} \lambda^{i}$ with $c_{i}=c_{i}(|\mathbf{P}|) \in \mathbb{Q}$. Given any tiling $\mathbf{T} \in \Omega_{\phi}$, let $\xi_{i}$ be the pattern equivariant 1-cochain on $\mathbf{T}$ defined by $\xi_{i}(T):=c_{i}(|T|)$ for each $T \in \mathbf{T}$.

Lemma 1. The cohomology classes $\left[\xi_{0}\right], \ldots,\left[\xi_{d-1}\right]$ are linearly independent in the pattern-equivariant cohomology of $\Omega_{\phi}$.

Proof. Suppose that $\alpha=\sum \alpha_{i} \xi_{i}=\delta(\beta)$, where $\beta$ is a pattern-equivariant 0-cochain and each $\alpha_{i}$ is rational. For large enough $k, \lambda^{k} L$ will be a return length between patches of size greater than twice the radius of $\beta$. Therefore, $\alpha$ applied to such a return patch of length $\lambda^{k+i} L$ must be zero for $i \geq 0$. But $\alpha$ applied to a patch depends only on the length of the patch, so $\alpha$ applied to any patch of length $\lambda^{k+i} L$ must be zero. As $\lambda^{d}+a_{d-1} \lambda^{d-1}+\cdots+a_{0}=0$, after division by $\lambda$, we have $a_{0} \lambda^{-1}=$ $-\left(\lambda^{d-1}+a_{d-1} \lambda^{d-2}+\cdots+a_{1}\right)$. Taking the $(k-i)$ th power of the last equation, we have $a_{0}^{k-i} \lambda^{i-k}$ as a polynomial in $\lambda$ with integer coefficients. Hence, $a_{0}^{k-i} L \lambda^{i}$ is an integer linear combination of $\lambda^{k} L, \lambda^{k+1} L, \ldots \lambda^{k+d-1} L$, so $\alpha_{i}$ must be zero.

If $\phi$ is a homological Pisot substitution, $\left\{\left[\xi_{0}\right], \ldots,\left[\xi_{d-1}\right]\right\}$ is a basis for the first cohomology of any tiling in $\Omega_{\phi}$. We use this to prove the Exact Regularity Property (Theorem 3) and its converse (Theorem 4).

Proof of Theorem [3. Let $\alpha$ be a pattern-equivariant 1-cochain on $\mathbf{T}$ (which we call an indicator cochain) that evaluates to 1 at each occurrence of the patch $\mathbf{P}$ and is identically zero away from $\mathbf{P}$. That is, choose a tile $T \in \mathbf{P}$ and define $\alpha$ by: if $T^{\prime}$ is any tile of $\mathbf{T}$, then $\alpha\left(T^{\prime}\right)=1$ if there is a $t \in \mathbb{R}$ such that $T+t=T^{\prime}$ and $\mathbf{P}+t \subset \mathbf{T}$, and $\alpha\left(T^{\prime}\right)=0$ otherwise. We must then have $\alpha=\delta(\beta)+\sum \alpha_{i} \xi_{i}$, where $\beta$ is a pattern-equivariant 0 -cochain of some radius $r$ and the coefficients $\alpha_{i}$ are rational. Let $L^{\prime}=\max \{r,|\mathbf{P}|\}$ and suppose that patch $\mathbf{Q} \subset \mathbf{T}$ has a vertex $x_{0}$ with $\left[x_{0}-L^{\prime}, x_{0}+L^{\prime}\right]$ contained in the support of $\mathbf{Q}$. Suppose also that $\mathbf{Q}+\tau \subset \mathbf{T}$. Since $\beta\left(x_{0}+\tau\right)=\beta\left(x_{0}\right)$, 
$\alpha\left(\left[x_{0}, x_{0}+\tau\right]\right)=\sum \alpha_{i} \xi_{i}\left(\left[x_{0}, x_{0}+\tau\right]\right)=\sum \alpha_{i} c_{i}(\tau)$. On the other hand, $\alpha\left(\left[x_{0}, x_{0}+\tau\right]\right)$ is the number of occurrences of $P$ between $x_{0}$ and $x_{0}+\tau$, this number being unambiguous (i.e., independent of choice of $T \in \mathbf{P}$ ) since $L^{\prime} \geq|\mathbf{P}|$.

If $L^{\prime \prime}$ is any return length, then for large $k, \lambda^{k+i} L^{\prime \prime}$ is a return length between patches of size greater than $L^{\prime}$ for all $i \geq 0$. Then $\sum \alpha_{i} \xi_{i}=\alpha-\delta(\beta)$ applied to a patch of length $\lambda^{k+i} L^{\prime \prime}$ yields an integer. However, $a_{0}^{k} L^{\prime \prime}$ is an integer linear combination of $\lambda^{k} L^{\prime \prime}, \lambda^{k+1} L^{\prime \prime}, \ldots, \lambda^{k+d-1} L^{\prime \prime}$. This implies that $\sum \alpha_{i} \xi_{i}$ applied to a patch of length $L^{\prime \prime}$ yields an integer divided by a power of $a_{0}$. Since $\sum \alpha_{i} \xi_{i}$ applied to any return word yields an integer divided by a power of $a_{0}$, and since $L \lambda^{i}$ is in the return lattice, all coefficients $\alpha_{i}$ must be integers divided by powers of $a_{0}$.

Proof of Theorem 4. Let $\alpha$ be an indicator cochain for a patch $\mathbf{P}$ (as in the proof above). There are then $\alpha_{i} \in \mathbb{Q}$ so that $\gamma:=\alpha-\sum \alpha_{i} \xi_{i}$ vanishes on chains of the form $\left[x_{0}, x_{0}+\tau\right]$ with $x_{0}$ a vertex in a patch $\mathbf{Q}$ of $\mathbf{T},\left[x_{0}-L^{\prime}, x_{0}+L^{\prime}\right]$ contained in the support of $\mathbf{Q}$, and $\mathbf{Q}+\tau \subset \mathbf{T}$. Let $\mathbf{Q}$ and $x_{0}$ be such a patch and vertex and define the 0 -cochain $g$ by $g(x):=\gamma\left(\left[x_{0}, x\right]\right)$ for each vertex $x$ of $\mathbf{T}$ (here $\left[x_{0}, x\right]$ means $-\left[x, x_{0}\right]$ for $x<x_{0}$, and we take $\left.g\left(x_{0}\right)=0\right)$. Then $g$ is pattern-equivariant and $\delta g=\gamma$. Since the indicator cochains span the pattern-equivariant 1-cochains, the $\left[\xi_{i}\right]$ form a basis for the first cohomology of $\mathbf{T}$.

Having established constraints on the number of occurrences of $\mathbf{P}$ for any return length $L$ (or at least $\lambda^{k} L$ for sufficiently large $k$ ), we establish constraints on the measure of the cylinder set of $\mathbf{P}$ with respect to the unique translation-invariant measure $\mu$ on $\Omega_{\phi}$. Let $S_{\mathbf{P}}$ be the set of all tilings in which the origin lies inside a $\mathbf{P}$ patch.

Proposition 1. Suppose that $\phi$ is a homological Pisot substitution of degree $d$ for which the tile and return lattices are the same, and let $p^{\prime}(\lambda)=d \lambda^{d-1}+\sum_{i=1}^{d-1} i a_{i} \lambda^{i-1}$ be the derivative of the minimal polynomial of the dilatation $\lambda$ of $\phi$, evaluated at $\lambda$. Then $\mu\left(S_{\mathbf{P}}\right)=\frac{q_{\mathbf{P}}(\lambda)}{a_{0}^{k} p^{\prime}(\lambda)}$, where $k$ is an integer and $q_{\mathbf{P}}(\lambda)$ is a polynomial in $\lambda$ with integer coefficients.

Proof. Let $\mathbf{P}$ be a patch based on the word $w=w_{1} \cdots w_{l}$. Given any patch $\mathbf{Q}$, let $S_{\mathbf{Q}}^{j}$ denote the (partial) cylinder set consisting of all tilings for which the origin not only lies in a $\mathbf{Q}$ patch, but in the $j$ th tile of the $\mathbf{Q}$ patch. We may then express $S_{\mathbf{P}}$ as a (measurably) disjoint union of sets of the form $S_{\mathbf{Q}}^{l+1}$ where $\mathbf{Q}$ has underlying word $u w_{i} v, u$ and $v$-letter words, $i \in\{1, \ldots, l\}$. For $\mathbf{Q}$ based on the word $u w_{i} v$ and $m$ such that the length of $\phi^{m}\left(w_{i}\right)$ is a return length, we take $L=\lambda^{m}\left|w_{i}\right|$. To compute the measure of $S_{\mathbf{Q}}^{l+1}$, we merely count how many times $\mathbf{Q}$ occurs in an interval of length $L \lambda^{k}$, divide by $\lambda^{k}$, take the limit as $k \rightarrow \infty$, and then multiply by $\left|w_{i}\right| / L$. To find 
the limit, we write $\lambda^{k}=\sum_{i=0}^{d-1} c_{k, i} \lambda^{i}$ and take the limit of $\sum \alpha_{i} c_{k, i} / \lambda^{k}$. The limit of $\vec{r}=\lim _{k \rightarrow \infty}\left(c_{k, 0}, \ldots, c_{k, d-1}\right)^{T} / \lambda^{k}$ is a right eigenvector of the companion matrix

$$
C=\left(\begin{array}{ccccc}
0 & 0 & \cdots & 0 & -a_{0} \\
1 & 0 & \cdots & 0 & -a_{1} \\
0 & 1 & \cdots & 0 & -a_{2} \\
\vdots & & \ddots & \vdots & \vdots \\
0 & 0 & \cdots & 1 & -a_{d-1}
\end{array}\right)
$$

normalized so that $\left(1, \lambda, \ldots, \lambda^{d-1}\right) \vec{r}=1$. This eigenvector is

$$
\vec{r}=\frac{1}{p^{\prime}(\lambda)}\left(\begin{array}{c}
\lambda^{d-1}+a_{d-1} \lambda^{d-2}+\cdots+a_{1} \\
\lambda^{d-2}+a_{d-1} \lambda^{d-3}+\cdots+a_{2} \\
\lambda^{d-3}+a_{d-1} \lambda^{d-4}+\cdots+a_{3} \\
\vdots \\
\lambda+a_{d-1} \\
1
\end{array}\right)
$$

Since each entry of $\vec{r}$ is a polynomial in $\lambda$ divided by $p^{\prime}(\lambda)$, and since each $\alpha_{i}$ is an integer divided by a power of $a_{0}$, the measure of $S_{\mathbf{Q}}^{l+1}$ is of the indicated form. It follows that $S_{\mathbf{P}}$ has the desired form as well.

Lemma 2. Let $\phi$ be a homological Pisot substitution of degree $d$ and dilatation $\lambda$ for which the tile and return lattices are the same. If some cylinder set in $\Omega_{\phi}$ has rational measure $n / m$, with $n$ and $m$ relatively prime, then $m$ divides $a_{0}^{k} \operatorname{gcd}\left(p^{\prime}(\lambda)\right)$ for some $k \in \mathbb{N}$, where $\operatorname{gcd}\left(p^{\prime}(\lambda)\right)$ is the greatest common divisor of the coefficients of $p^{\prime}(\lambda)$ and $a_{0}$ is the constant coefficient of $\lambda$.

Proof. If $\frac{q_{\mathbf{P}}(\lambda)}{a_{0}^{k} p^{\prime}(\lambda)}=\frac{n}{m}$, then $m q_{\mathbf{P}}(\lambda)=a_{0}^{k} p^{\prime}(\lambda) n$. But this means that $m$ divides every coefficient of $a_{0}^{k} p^{\prime}(\lambda)$, and so divides $a_{0}^{k} \operatorname{gcd}\left(p^{\prime}(\lambda)\right)$.

Theorem 5 is an immediate corollary of Lemma 2 .

\section{Substitutions with $d=1$}

If $d=1$, then the dilatation is an integer $N$, and the norm is $N$ itself. Under these circumstances, we can replace the substitution with an 'equivalent' substitution that has constant length using the following technique.

First scale the left Perron-Frobenius eigenvector so that all entries are integers whose greatest common factor is one. That is, choose all of the tiles to have integral length. Then subdivide each tile into smaller pieces, each of length one. The substitution, 
written in terms of these pieces, will have constant length $N$. If the resulting substitution has return lattice $h \mathbb{Z}$, with $h>1$, we can regroup these pieces into tiles of size $h$ and rescale by $h$. The resulting substitution has constant length, with both the tile and return lattices equaling $\mathbb{Z}$, and is called the pure core of the original substitution. This new substitution and the original substitution have conjugate tiling flows, hence their coincidence ranks are the same and one is homological Pisot if and only if the other is.

Consider the substitution $\phi$ of constant length $N$ acting on the alphabet $\mathcal{A}_{\phi}$. We say that two words $w_{1} w_{2} w_{3} \ldots$ and $v_{1} v_{2} v_{3} \ldots$ are coincident if there exists a $k$ such that $w_{k}=v_{k}$. We say that the letters $a$ and $b$ in $\mathcal{A}_{\phi}$ are eventually coincident if there exist a $k$ and $n$ such that $\phi^{n}(a)_{k}=\phi^{n}(b)_{k}$, where $\phi^{n}(a)_{k}$ denotes the $k$ th letter of $\phi^{n}(a)$. We say that the letters $a$ and $b$ are strongly coincident if for each $n \geq 0$ and each $i \in\left\{1, \ldots, N^{n}\right\}$, the $i$ th letters of $\phi^{n}(a)$ and $\phi^{n}(b)$ are eventually coincident.

Proposition 2. Let $\phi$ be a substitution of constant length $N$ and let $\phi^{\prime}$ be the substitution obtained by identifying the letters in $\mathcal{A}_{\phi}$ that are strongly coincident. If $\phi$ is a homological Pisot substitution, then so is $\phi^{\prime}$.

Proof. If $\phi$ is a homological Pisot substitution, then $\phi$ has the Exact Regularity Property by Theorem 3 . Since every patch $\mathbf{P}^{\prime}$ in a tiling of $\Omega_{\phi^{\prime}}$ corresponds to a finite set of patches $\left\{\mathbf{P}_{1}, \ldots, \mathbf{P}_{n}\right\}$ in $\Omega_{\phi}$, and since each of these patches $\mathbf{P}_{i}$ is governed by the ERP, we will show that $\mathbf{P}^{\prime}$ is governed by the ERP, implying that $\phi^{\prime}$ is a homological Pisot substitution.

The patches $\mathbf{P}_{i}$ exhibit the ERP with different lengths $L_{i}^{\prime}$. Let $L^{\prime}=\max \left\{L_{i}^{\prime}\right\}$. Pick any patch $\mathbf{S}^{\prime}$ in $\Omega_{\phi^{\prime}}$ with a vertex that is a distance at least $L^{\prime}$ from each end. $\mathbf{S}$ corresponds to a finite set $\left\{\mathbf{S}_{1}, \ldots, \mathbf{S}_{m}\right\}$ of patches in $\Omega_{\phi}$, each with a vertex of distance at least $L^{\prime}$ from the end. By the ERP for each $\mathbf{P}_{i}$, between any two successive $\mathbf{S}_{i}$ 's, there are exactly the right number of $\mathbf{P}_{1}$ 's, the right number of $\mathbf{P}_{2}$ 's, etc, so between any two $\mathbf{S}^{\prime}$ 's that correspond to the same $\mathbf{S}_{i}$, there are exactly the right number of $\mathbf{P}^{\prime \prime}$ s. The problem is that different occurrences of $\mathbf{S}^{\prime}$ may correspond to different $\mathbf{S}_{i}$ 's.

The discrepancy in how many extra $\mathbf{P}^{\prime}$ s occur between an $\mathbf{S}_{i}^{\prime}$ and an $\mathbf{S}_{j}^{\prime}$ depends only on $i$ and $j$, since the discrepancy between any two $\mathbf{S}_{i}^{\prime}$ s, or any two $\mathbf{S}_{j}^{\prime}$ s, is zero. In particular, there exist numbers $z_{1}, \ldots, z_{m}$ so that there are exactly $z_{i}-z_{j}$ extra $\mathbf{P}^{\prime}$ 's between any $\mathbf{S}_{i}^{\prime}$ and any $\mathbf{S}_{j}^{\prime}$. Order the images such that $z_{1} \geq z_{2} \geq \ldots \geq z_{m}$. Since $\phi$ is a primitive substitution, $\Omega_{\phi}$ is repetitive, so every sufficiently large patch (say, of diameter $D$ ) contains at least one copy of $\mathbf{S}_{1}$ and at least one copy of $\mathbf{S}_{m}$.

Now let $\mathbf{Q}^{\prime}$ be any patch of size greater than $D$. Each patch $\mathbf{Q}_{i}$ in $\Omega_{\phi}$ that maps to $\mathbf{Q}^{\prime}$ must contain an $\mathbf{S}_{1}$ and an $\mathbf{S}_{m}$. The images of the $\mathbf{S}$ patches with maximal and minimal values of $z_{i}$ can be identified, in $\mathbf{Q}^{\prime}$, by the $z_{1}-z_{m}$ extra (or missing) $\mathbf{P}^{\prime \prime}$ s that occur between them, which can only occur between an $\mathbf{S}_{i}^{\prime}$ with $z_{i}=z_{1}$ and an $\mathbf{S}_{j}^{\prime}$ with 
$z_{j}=z_{m}$. This means that the locations of patches $\mathbf{S}_{i}^{\prime}$ with $z_{i}=z_{1}$ line up exactly for any two patches $\mathbf{Q}^{\prime}$, so the number of $\mathbf{P}^{\prime \prime}$ s between any two $\mathbf{Q}^{\prime}$ 's is exactly correct.

We define a stable pair as two letters in $\mathcal{A}_{\phi}$ which are not eventually coincident and extend this idea to arbitrary collections of letters: a stable $m$-tuple is a collection $\left\{a_{1}, a_{2}, \ldots, a_{m}\right\}$ of letters from $\mathcal{A}_{\phi}$ such that for all $n$ and $0 \leq k \leq N^{n}$, $\left\{\phi^{n}\left(a_{1}\right)_{k}, \phi^{n}\left(a_{2}\right)_{k}, \ldots, \phi^{n}\left(a_{m}\right)_{k}\right\}$ has cardinality $m$. For constant length substitutions, the coincidence rank, $c r$, is the minimum cardinality of $\left\{\phi^{n}\left(a_{1}\right)_{k}, \ldots, \phi^{n}\left(a_{\left|\mathcal{A}_{\phi}\right|}\right)_{k}\right\}$ for $0 \leq k \leq N^{n}, n \in \mathbb{Z}$, see [BBK]. Hence $m=c r$ is the maximal cardinality of a stable $m$-tuple and it follows that the coincidence ranks of $\phi$ and $\phi^{\prime}$ are the same. Therefore, to obtain constraints on $\mathrm{cr}$ for homological Pisot substitutions, it suffices to consider substitutions for which no two letters are strongly coincident.

Proposition 3. If $\phi$ is a homological Pisot substitution of constant length $N$ and coincidence rank cr, with no strongly coincident pairs of letters, then there exists a set of letters whose cylinder sets have total measure $1 / \mathrm{cr}$.

Proof. For sufficiently large $n$, there exists a $k$ such that $\left\{\phi^{n}\left(a_{i}\right)_{k}\right\}, i \in\left\{1, \ldots,\left|\mathcal{A}_{\phi}\right|\right\}$ consists of exactly $\mathrm{cr}$ elements. (Asymptotically, the number of such $k$, divided by $N^{n}$, approaches 1, [De] $)$. Let $n$ and $k$ be as described above, and let the set $\left\{\phi^{n}\left(a_{i}\right)_{k}\right\}$ be given by $\left\{b_{1}, b_{2}, \ldots, b_{c r}\right\}$. This set is a stable $c r$-tuple. There are then tilings $\mathbf{T}^{i} \in \Omega_{\phi}, i=1, \ldots, c r$, and an $m \in \mathbb{N}$, so that $\Phi^{m}\left(\mathbf{T}^{i}\right)=\mathbf{T}^{i}$ and 0 is the left endpoint of a tile of type $b_{i}$ in $\mathbf{T}^{i}$. Partition $\mathcal{A}_{\phi}$ into sets $B_{j}:=\left\{a: \phi^{n}(a)_{k}=b_{j}\right\}, j \in\{1, \ldots, c r\}$. Let $C_{j}$ be the union of the cylinder sets corresponding to letters in $B_{j} ; C_{j}:=\{\mathbf{T}$ : $0 \in T_{0}, T_{0} \in \mathbf{T}, T_{0}$ of type $a$ for some $\left.a \in B_{j}\right\}$. For fixed $j$ and for each $t \in \mathbb{R}^{+} \backslash \mathbb{N}$, exactly one of the tilings $\mathbf{T}^{i}$ is in $C_{j}$. Thus $1=\lim _{\tau \rightarrow \infty} \frac{1}{\tau} \int_{0}^{\tau} \sum_{j=1}^{c r} \chi_{C_{j}}\left(\mathbf{T}^{j}-t\right) d t=$ $\sum_{j=1}^{c r} \lim _{\tau \rightarrow \infty} \frac{1}{\tau} \int_{0}^{\tau} \chi_{C_{j}}\left(\mathbf{T}^{j}-t\right) d t=\operatorname{cr} \mu\left(C_{j}\right)$, by unique ergodicity of the tiling flow. Thus, for each $j$, the measure of the union of the cylinder sets of the elements of $B_{j}$ is $1 / c r$.

By Theorem 5, this implies that $c r$ divides a power of $N$, thereby proving Theorem 2 , namely that the Coincidence Rank Conjecture holds for $d=1$.

We finish this section with the proof of Theorem 6, namely that a homological Pisot substitution with $d=1$ cannot have $c r=2$.

Proof. If $N$ is odd, this follows from Theorem [2. Suppose, then, that $N$ is even and $c r=2$. We will prove that $\operatorname{dim}\left(H^{1}\right)>1$. Every element of $\mathcal{A}_{\phi}$ must be a member of a stable pair by primitivity and the fact that there exist $n, k$ such that $\left\{\phi^{n}\left(a_{1}\right)_{k}, \phi^{n}\left(a_{2}\right)_{k}, \ldots, \phi^{n}\left(a_{\left|\mathcal{A}_{\phi}\right|}\right)_{k}\right\}$ has cardinality $c r$. Suppose that $a$ is in a stable pair with $a^{\prime}$ and $a^{\prime \prime}, a^{\prime} \neq a^{\prime \prime}$. Then $a^{\prime}$ and $a^{\prime \prime}$ must be strongly coincident, or 
$c r=2$ is violated. By identifying strongly coincident letters, and recalling the results of Proposition 2, it suffices to consider the case of an alphabet of $2 m$ letters $\left\{a_{1}, \ldots, a_{m}, a_{1}^{\prime}, \ldots, a_{m}^{\prime}\right\}$, where $a_{i}$ and $a_{i}^{\prime}$ form a stable pair.

Ordering the columns and rows of the abelianization by $a_{1}, \ldots, a_{m}, a_{1}^{\prime}, \ldots, a_{m}^{\prime}$, the matrix takes the form $\mathbf{A}=\left(\begin{array}{ll}A & B \\ B & A\end{array}\right)$, where $A$ and $B$ are $m \times m$ matrices, In particular, the trace of $\mathbf{A}$ is even.

The space $G_{0}^{E R}$ appearing in (1) is the direct limit under substitution of the graph obtained from all the edges $v_{i j}$ that describe two-letter words $a_{i} a_{j}$ and likewise edges $v_{i^{\prime} j}, v_{i j^{\prime}}$ and $v_{i^{\prime} j^{\prime}}$.

The symmetry that exchanges each $a_{i}$ for $a_{i}^{\prime}$ maps the components of $G_{0}^{E R}$ to each other. If any component is mapped to itself, then there exists a loop in $G_{0}^{E R}$, implying that the dimension of $\check{H}^{1}\left(\Omega_{\phi}\right)$ exceeds $d$. To see this, suppose that $v_{i j}$ and $v_{i^{\prime} j^{\prime}}$ are in the same component. Then there exists a path in the graph connecting these two edges. Letting $v_{i j}=v_{i_{1} j_{1}}$, this path may be labeled as $v_{i_{1} j_{1}}, v_{i_{2} j_{1}}, v_{i_{2} j_{2}}, v_{i_{3} j_{2}}, \ldots, v_{i^{\prime} j^{\prime}}$. Since the existence of the word $a_{i} a_{j}$ in the substitution implies the appearance of $a_{i}^{\prime} a_{j}^{\prime}$ as well, the path $v_{i^{\prime} j^{\prime}}=v_{i_{1}^{\prime} j_{1}^{\prime}}, v_{i_{2}^{\prime} j_{1}^{\prime}}, v_{i_{2}^{\prime} j_{2}^{\prime}}, \ldots, v_{i j}$ exists also, creating a loop in $G_{0}^{E R}$.

If no component is mapped to itself, then there are an even number of components in $G_{0}^{E R}$, which come in symmetric pairs. Since $\phi$ is a homological Pisot substitution, the dimension of $\check{H}^{1}\left(\Omega_{\phi}\right)$ is one. This implies that the nonzero eigenvalues of $\mathbf{A}^{T}$ must be $N$ (with multiplicity one) and 1 (with odd multiplicity). This is impossible, since $N$ is even and the trace of $\mathbf{A}^{T}$ is even.

\section{EXAMPLES}

In this section we provide examples of homological Pisot substitutions with $\mathrm{cr}>1$. Specifically, all examples have $c r=3$ and all dilatations have norm divisible by 3 . In each case we first construct a homological Pisot substitution with $\mathrm{cr}=1$ and alphabet $\mathcal{A}_{1}=\{A, B, \ldots\}$, and then construct another homological Pisot substitution with alphabet $\mathcal{A}_{2}=\left\{a_{1}, a_{2}, a_{3}, b_{1}, b_{2}, b_{3}, \ldots\right\}$ that is a triple cover of the first, and hence has $c r=3$.

\section{Example 1: $d=1$}

We take a 2-letter alphabet $\mathcal{A}_{1}=\{A, B\}$ and a substitution

$$
\begin{aligned}
& \phi_{1}(A)=A B A B A A A B A \\
& \phi_{1}(B)=B A A A B A A B A
\end{aligned} \quad \text { with abelianization } \quad\left(\begin{array}{ll}
6 & 3 \\
6 & 3
\end{array}\right)
$$

Since all substituted letters end in $A$, the first cohomology of $\Omega_{\phi_{1}}$ is the direct limit of the abelianization (see [BD2]) and has dimension one. 
For $\mathcal{A}_{2}$, we associate three letters to each letter in $\mathcal{A}_{1}$, and a permutation of those three letters to each transition $A A, A B, B A$ or $B B$. (Actually, there are no transitions $B B$, but we include a permutation as a demonstration of the method.) Specifically, to the transition $A A$ we associate $a_{1} a_{3}, a_{3} a_{1}$ and $a_{2} a_{2}$. To $A B$ we associate $a_{1} b_{2}, a_{2} b_{1}$ and $a_{3} b_{3}$. To $B A$ we associate $b_{1} a_{3}, b_{3} a_{1}$ and $b_{2} a_{2}$. To $B B$ we associate $b_{1} b_{2}, b_{2} b_{1}$, and $b_{3} b_{3}$. In short, we are allowing transitions $\left\{a_{1}\right.$ or $\left.b_{1}\right\}\left\{a_{3}\right.$ or $\left.b_{2}\right\},\left\{a_{2}\right.$ or $\left.b_{2}\right\}\left\{a_{2}\right.$ or $\left.b_{1}\right\}$, and $\left\{a_{3}\right.$ or $\left.b_{3}\right\}\left\{a_{1}\right.$ or $\left.b_{3}\right\}$.

Note that there are exactly three words in the alphabet $\mathcal{A}_{2}$ associated to each word in the alphabet $\mathcal{A}_{1}$. There are three choices on what the first letter should be, and the rest of the word is determined by the allowed transitions. These three words disagree at each point.

The substitution that generates these words is

$$
\begin{aligned}
& \phi_{2}\left(a_{1}\right)=a_{1} b_{2} a_{2} b_{1} a_{3} a_{1} a_{3} b_{3} a_{1} \\
& \phi_{2}\left(a_{2}\right)=a_{2} b_{1} a_{3} b_{3} a_{1} a_{3} a_{1} b_{2} a_{2} \\
& \phi_{2}\left(a_{3}\right)=a_{3} b_{3} a_{1} b_{2} a_{2} a_{2} a_{2} b_{1} a_{3} \\
& \phi_{2}\left(b_{1}\right)=b_{1} a_{3} a_{1} a_{3} b_{3} a_{1} a_{3} b_{3} a_{1} \\
& \phi_{2}\left(b_{2}\right)=b_{2} a_{2} a_{2} a_{2} b_{1} a_{3} a_{1} b_{2} a_{2} \\
& \phi_{2}\left(b_{3}\right)=b_{3} a_{1} a_{3} a_{1} b_{2} a_{2} a_{2} b_{1} a_{3}
\end{aligned} \quad \text { with abel- } \quad \text { ianization } \quad\left(\begin{array}{cccccc}
3 & 2 & 1 & 3 & 1 & 2 \\
1 & 2 & 3 & 0 & 4 & 2 \\
2 & 2 & 2 & 3 & 1 & 2 \\
1 & 1 & 1 & 1 & 1 & 1 \\
1 & 1 & 1 & 0 & 2 & 1 \\
1 & 1 & 1 & 2 & 0 & 1
\end{array}\right)
$$

Note that for each $x_{i}$ (where $x=a$ or $b$ ), $\phi\left(x_{i}\right)$ begins with $x_{i}$ and ends with $a_{i}$. This ensures that substituted words will have the same allowed transitions as the original words.

The abelianization has eigenvalues $9,1,1,0,0,0$. Since the direct limit of the transition graph has 3 components, each of which is contractible, this implies that $\check{H}^{1}\left(\Omega_{\phi}, \mathbb{Q}\right)$ is 1-dimensional.

Example 2: $d=2$

We take $\mathcal{A}_{1}$ and $\mathcal{A}_{2}$ as before, only now $\phi_{1}$ is a substitution of degree 2 , with abelianization $\left(\begin{array}{ll}9 & 6 \\ 6 & 3\end{array}\right)$ and dilatation $6+3 \sqrt{5}$ :

$$
\begin{aligned}
& \phi_{1}(A)=A B A B A A A B A B A B A B A \\
& \phi_{1}(B)=B A A A B A A B A
\end{aligned}
$$

This is just like the $d=1$ example, only with a $B A B A B A$ suffix applied to $\phi_{1}(A)$. Construct $\phi_{2}$ similar to (2). This suffix induces a trivial permutation, and so continues to allow us to have $\phi_{2}\left(x_{i}\right)$ begin with $x_{i}$ and end with $a_{i}$. The abelianization for $\phi_{2}$ is 
now

$$
\left(\begin{array}{llllll}
4 & 3 & 2 & 3 & 1 & 2 \\
2 & 3 & 4 & 0 & 4 & 2 \\
3 & 3 & 3 & 3 & 1 & 2 \\
2 & 2 & 2 & 1 & 1 & 1 \\
2 & 2 & 2 & 0 & 2 & 1 \\
2 & 2 & 2 & 2 & 0 & 1
\end{array}\right)
$$

and has eigenvalues $6+3 \sqrt{5}, 6-3 \sqrt{5}, 1,1,0,0$. As before, the direct limit of the transition graph has 3 components, each of which is contractible, so $\check{H}^{1}\left(\Omega_{\phi}, \mathbb{Q}\right)$ is 2 dimensional.

Example 3: $d=3$

We now take $\mathcal{A}_{1}=\{A, B, C\}$, with substitution

$$
\begin{aligned}
& \phi_{1}(A)=A B A B A A A B A(C B A)^{6}\left(B A^{3}\right)^{3} \\
& \phi_{1}(B)=B A A A B A A B A(C B A)^{3}\left(C B A^{3}\right)^{3} \\
& \phi_{1}(C)=(C B A)^{3}\left(B A^{3}\right)^{3} .
\end{aligned}
$$

with dilatation $\lambda=3 \theta^{4}$ where $\theta$ is the tribonacci dilatation, i.e., the leading root of $\theta^{3}=\theta^{2}+\theta+1$. The eigenvalues of the abelianization of $\phi_{1}$ are $\lambda$ and its algebraic conjugates. It is not hard to see that $\operatorname{dim}\left(\check{H}^{1}\left(\Omega_{\phi}, \mathbb{Q}\right)\right)=3$, and that the coincidence rank of $\phi_{1}$ is 1 .

As far as $\phi_{2}$ and permutations of $\{1,2,3\}$ go, $C$ is merely a spectator, with transitions ending in $C$ having a trivial permutation and transitions beginning with $c_{i}$ having the same permutations as transitions beginning with $a_{i}$ or $b_{i}$. Specifically, the allowed transitions for $\Omega_{\phi_{2}}$ are $\left\{a_{1}\right.$ or $b_{1}$ or $\left.c_{1}\right\}\left\{a_{3}\right.$ or $b_{2}$ or $\left.c_{1}\right\}$, $\left\{a_{2}\right.$ or $b_{2}$ or $\left.c_{2}\right\}\left\{a_{2}\right.$ or $b_{1}$ or $\left.c_{2}\right\}$, and $\left\{a_{3}\right.$ or $b_{3}$ or $\left.c_{3}\right\}\left\{a_{1}\right.$ or $b_{3}$ or $\left.c_{3}\right\}$.

The terms $(C B A)^{3},\left(B A^{3}\right)^{3}$ and $\left(C B A^{3}\right)^{3}$ induce trivial permutations of $\{1,2,3\}$ and give equal populations of $x_{1}, x_{2}, x_{3}$ to $\phi_{2}$ of any letter. They are special cases of the "padding" discussed below in Example 4.

The nonzero eigenvalues of the abelianization of $\phi_{2}$ are $1,1, \lambda$, and the algebraic conjugates of $\lambda$. Since the eventual range of the transition graph of $\phi_{2}$ has 3 contractible components, $\operatorname{dim}\left(\check{H}^{1}\left(\Omega_{\phi_{2}}\right)\right)=\operatorname{dim}\left(\check{H}^{1}\left(\Omega_{\phi_{1}}\right)\right)=3$.

\section{Example 4: Arbitrary $d$}

The same tricks can be used to create examples of arbitrary degree and $\mathrm{cr}=3$.

Start with any primitive $d \times d$ Pisot matrix $M_{0}$ with odd determinant. Since the determinant is $1(\bmod 2)$, some power $M_{0}^{k}$ is equal to the identity $(\bmod 2)$, and we can assume that $k$ is big enough for all but one of the eigenvalues of $M_{0}^{k}$ to be smaller than $1 / 3$. Let $M_{1}=3 M_{0}^{k}$ be a new Pisot abelianization on $d$ letters $\mathrm{A}, \mathrm{B}, \ldots, \mathrm{Z}$, which 
is the abelianization of a substitution of the form

$$
\begin{aligned}
\phi_{1}(A) & =A B A B A A A B A \text { plus padding, } \\
\phi_{1}(B) & =B A A A B A A B A \text { plus padding, } \\
\phi_{1} \text { (any other letter) } & =\text { nothing but padding, }
\end{aligned}
$$

where "padding" means a product of words, each of the general form $\left(V B^{\text {odd }} W A^{\text {odd }} Y\right)^{3}$, where $V, W$ and $Y$ are arbitrary words in the letters other than $A$ and $B$. We can always choose the padding such that $\phi_{1}$ of any letter begins with that letter and ends in $A$.

For $\Omega_{\phi_{2}}$, the allowed transitions are $\left\{\right.$ any $\left.x_{1}\right\}\left\{a_{3}\right.$ or $b_{2}$ or any other $\left.y_{1}\right\},\left\{\right.$ any $\left.x_{2}\right\}\left\{a_{2}\right.$ or $b_{1}$ or any other $\left.y_{2}\right\}$, and $\left\{\right.$ any $\left.x_{3}\right\}\left\{a_{1}\right.$ or $b_{3}$ or any other $\left.y_{3}\right\}$. With these choices, padding yields trivial permutations, and we can pick $\phi_{2}\left(x_{i}\right)$ to begin with $x_{i}$ and end with $a_{i}$.

The abelianization $M_{2}$ for $\phi_{2}$ can be viewed as a collection of $3 \times 3$ blocks, one for each matrix element of the abelianization for $\phi_{1}$. All blocks other than the $A A, A B$, $B A$ and $B B$ blocks are multiples of $\left(\begin{array}{lll}1 & 1 & 1 \\ 1 & 1 & 1 \\ 1 & 1 & 1\end{array}\right)$. This is because "padding" yields equal numbers of $x_{1}, x_{2}$ and $x_{3}$. The $A A, A B, B A$ and $B B$ blocks are the same as in our $d=1$ example, plus multiples of $\left(\begin{array}{lll}1 & 1 & 1 \\ 1 & 1 & 1 \\ 1 & 1 & 1\end{array}\right)$.

The populations of $\phi_{2}\left(a_{1}\right)$ and $\phi_{2}\left(a_{3}\right)$ add up to twice the population of $\phi_{2}\left(a_{2}\right)$, and the populations of $\phi_{2}\left(b_{1}\right)$ and $\phi_{2}\left(b_{2}\right)$ add up to twice the population of $\phi_{2}\left(b_{3}\right)$. For $X \neq A$ or $B$, the populations of $\phi_{2}\left(x_{1}\right), \phi_{2}\left(x_{2}\right)$ and $\phi_{2}\left(x_{3}\right)$ are all the same. This yields $2 d-2$ vectors in the kernel of $M_{2}$, so the rank of $M_{2}$ is at most $d+2$. However, every eigenvalue of $M_{1}$ is also an eigenvalue of $M_{2}$. Since the eventual range of the transition graph of $\phi_{2}$ has three components, 1 must be an eigenvalue of $M_{2}$ with multiplicity 2 . Together, these imply that $\operatorname{dim}\left(\check{H}^{1}\left(\Omega_{\phi_{2}}\right)\right)=d$.

Acknowledgments. The work of L.S. is partially supported by the National Science Foundation. M.B. and H.B. thank the Mathematisches Forschungsinstitut Oberwolfach for its hospitality during the Research in Pairs Programme January 11-24, 2009, and in this context we are also grateful to Sonja Štimac. H.B. thanks Delft University of Technology as well for its support during the summer of 2009.

\section{REFERENCES}

[A] J. Auslander, Minimal flows and their extensions, North-Holland Mathematical Studies, vol. 153, North-Holland, Amsterdam, New York, Oxford, and Tokyo, (1988).

[AP] J. E. Anderson and I. F. Putnam, Topological invariants for substitution tilings and their associated $C^{*}$-algebras, Ergodic Theory $\&$ Dynamical Systems 18 (1998), 509-537. 
[BBK] V. Baker, M. Barge and J. Kwapisz, Geometric realization and coincidence for reducible nonunimodular Pisot tiling spaces with an application to $\beta$-shifts, J. Instit. Fourier. 56 (7) (2006), 2213-2248.

[BD1] M. Barge and B. Diamond, A complete invariant for the topology of one-dimensional substitution tiling spaces, Ergodic Theory $\&$ Dynamical Systems 21 (2001), 1333-1358.

[BD2] M. Barge and B. Diamond, Coincidence for substitutions of Pisot type, Bulletin de la Société Mathématique de France 130 (2002), 619-626.

[BD3] M. Barge and B. Diamond, Cohomology in one-dimensional substitution tiling spaces, Proc. Amer. Math. Soc. 136 (6) (2008), 2183-2191.

[BK] M. Barge and J. Kwapisz, Geometric theory of unimodular Pisot substitutions, Amer J. Math. 128 (2006), 1219-1282.

[BS] V. Berthé and A. Siegel, Tilings associated with beta-numeration and substitutions, Integers: electronic journal of combinatorial number theory 5 (2005), A02.

[BSw] M. Barge and R. Swanson, Rigidity in one-dimensional tiling spaces, Top. and its Appl. 157 (17) (2007), 3095-3099.

[BT] E. Bombieri and J. E. Taylor, Which distributions of matter diffract? An initial investigation, J. Physique 47 (7, suppl. Colloq. C3) C3-19-C3-28, International workshop on aperiodic crystals (les Houches) (1986).

[CS] V. Canterini and A. Siegel, Geometric representation of substitutions of Pisot type, Trans. Amer. Math. Soc. 353 (2001), 5121-5144.

[De] F. M. Dekking, The spectrum of dynamical systems arising from substitutions of constant length, Z. Wahrscheinlichkeitstheorie verw. Gebiete 41 (1978), 221-239.

[Dw] S. Dworkin, Spectral theory and x-ray diffraction, J. Math. Phys. 34 (1993), 2964-2967.

[HS] M. Hollander and B. Solomyak, Two-symbol Pisot substitutions have pure discrete spectrum, Ergodic Theory \& Dynamical Systems 23 (2003), 533-540.

[LMS] J.-Y. Lee, R. V. Moody and B. Solomyak, Pure point dynamical and diffraction spectra, Annales Henri Poincaré 3 (2002), 1003-1018.

[M] B. Mossé, Puissances de mots et reconnaissabilité des points fixes d'une substitution, Theoretical Computer Science 99 (1992), 327-334.

[IR1] S. Ito and H. Rao, Atomic surfaces, tilings and coincidences I. Irreducible case, Israel J. Math. 153 (2006), 129-156.

[IR2] S. Ito and H. Rao, Atomic surfaces, tilings and coincidences II. Reducible case, Ann. Inst. Fourier, Grenoble 56 (6) (2006), 2285-2313.

[Kel] J. Kellendonk, Pattern-equivariant functions and cohomology, J. Phys. A. 36 (2003), 1-8.

[KP] J. Kellendonk and I. Putnam, The Ruelle-Sullivan map for $\mathbb{R}^{n}$-actions, Math. Ann. 334 (2006), 693-711.

[Sa1] L. Sadun, Pattern-equivariant cohomology with integer coefficients. Ergodic Theory E Dynamical Systems 27 (2007), 1991-1998.

[Sa2] L. Sadun, Exact regularity and the cohomology of tiling spaces, arXiv:math/1004.2281 〈http://arxiv.org/abs/1004.2281v1〉, 1-15.

[So1] B. Solomyak, Eigenfunctions for substitution tiling systems, Advanced Studies in Pure Mathematics, 49 (2007), 433-454.

[So2] B. Solomyak, Nonperiodicity implies unique composition for self-similar translationally finite tilings, Discrete and Computational Geometry 20 (2) (1998), 265-279. 
Department of Mathematics, Montana State University, Bozeman, MT 59717, USA barge@math.montana.edu

Department of Mathematics, University of Surrey, Guildford, Surrey GU2 7XH, UK h.bruin@surrey.ac.uk

Department of Mathematics, University of Arizona, Tucson, AZ 85724 USA

ljones@math.arizona.edu

Department of Mathematics, University of Texas, Austin, TX 78712, USA

sadun@math.utexas.edu 